\title{
Une réalité institutionnelle du tri en médecine ou comment faire au mieux?
}

\section{An institutional reality sorting medicine, how do the best? \\ K. Bréhaux (Docteur de science politique de l'Institut d'Études Politiques de Paris, Master 2 de Philosophie)}

\author{
Reims, France
}

Disponible sur Internet le 12 juin 2015

\section{MOTS CLÉS \\ Soin ; \\ Logique utilitariste ; \\ Libéralisme ; \\ Égalitarisme ; \\ Tri}

\begin{abstract}
Résumé Comment peut-on faire du soin aujourd'hui? Comment substituer une logique égalitariste à une logique utilitariste? Le triage est à la fois une promesse et une épreuve: un fondement de l'identité et de la profession médicale moderne et une intrusion menaçante des contraintes économiques et politiques dans la décision médicale. Comment maintenir une offre de soins efficace avec nos fondements libéraux et sociétaux? Ce double mouvement économique libéral-égalitariste fait du triage le pivot de débats autour des pratiques médicales. Cela pose aussi la question des critères éthiques et médicaux qui définissent l'accès des patients individuels à des ressources rares. Ce devoir de secours mobilise les citoyens, autour de valeurs et entre dans le champ des questions politiques. L'évaluation de la performance d'un système de santé et de ses moyens où il existe une pluralité de valeurs peut tromper la rationalité utilitariste. Nous superposons aux questions politiques du vivre ensemble les questions de principes qui se tranchent au cas par cas.

(c) 2015 Elsevier Masson SAS. Tous droits réservés.
\end{abstract}

Summary How can we care today? How to substitute an egalitarian logic to an utilitarian logic? Triage is both a promise and a challenge: a basis for identity and modern medical profession and a threatening intrusion of economic and political constraints in medical decision. How to maintain an effective system care with our liberal and social foundations? This double-liberal egalitarian economic movement is central to sorting debates about medical practices. This also raises the question of ethical and medical criteria that define access for individual patients to

\section{KEYWORDS \\ Care; \\ Utilitarian logic; \\ Liberalism; \\ Egalitarianism; \\ Sorting}

Adresse e-mail : karinebrehaux@orange.fr 
scarce resources. This duty of support mobilizes citizens around values and enters the field of political issues. The evaluation of the performance of a health system and its resources where there is a plurality of values can fool the utilitarian rationality. We superimpose political issues of living together issues of principles that settle case by case.

(c) 2015 Elsevier Masson SAS. All rights reserved.

\section{Introduction}

Face à une réalité du tri en médecine, comment faire au mieux pour garantir un accès des soins de qualité pour tous? Premier constat: comment en sommes-nous arrivés là? Comment maintenir une offre de soins cohérente avec nos fondements libéraux et sociétaux? Comment peut-on faire du soin aujourd'hui? Un sentiment de grand écart existe entre une pratique médicale tiraillée entre la réduction des inégalités sociales de santé (accès aux soins des plus démunis) et celle d'une médecine qui va jusqu'à «l'esthétique». Face à une réalité institutionnelle du tri en médecine, comment faire au mieux pour garantir un accès à des soins de qualité pour tous?

Notre postulat ici est le suivant: l'éthique du triage (conception moderne et pratique de la médecine issue de la médecine militaire) bien que décriée parce qu'utilitariste (et nous en donnerons les raisons) reste inscrite dans notre champ institutionnel de manière cachée parce que dépréciée dont seuls quelques avatars ou exceptions révèlent cette existence cachée. En témoigne, la littérature internationale croissante autour des notions de «qualité de vie» «futilité des traitements médicaux» «inutilité des traitements». Notre hypothèse de travail est de poser les questions éthiques au sein du système médicoéconomique. À travers des exemples de questions relevant de la santé publique, nous explorons cette dimension utilitariste inhérente au système aux prises avec des aspirations égalitaristes et libérales.

\section{L'éthique du triage comme un mode de gestion des politiques de soins aujourd'hui : sommes-nous en période de guerre?}

«Bien sur le triage est nécessaire. Il fait partie de la guerre. Il fait partie de ton job de médecin» Jon Kerstetter, «River Teeth: A journal of non fiction narrative», printemps 2012, volume 13 , numéro 2 , page 63 [1].

\section{Le tri comme expression d'une gestion économique et d'une philosophie utilitariste}

En période de guerre, il faut décider dans l'urgence d'envoyer à leur mort des soldats en attente pour se consacrer par un choix calculé, à un bien d'ordre supérieur, mesuré par le nombre de survivants au combat. Le triage militaire tient non seulement à la technicité et à la gravité des décisions à prendre mais aussi au fait que le contexte de combat rend ses décisions nécessaires. L'éthique utilitariste aux fondements de la médecine militaire, le fait de trier, de sauver des vies par le sacrifice de quelques-unes, quelle que soit la sophistication des protocoles de décision et de classement, revient à obliger les médecins à accomplir des tâches qui partagent la brutalité et l'horreur de la guerre. Transposer dans un contexte actuel de paix relative, hors du monde militaire, le triage au sens général de l'opération par laquelle un médecin classe les patients par ordre de priorité, prend des formes dramatiques mais aussi lourdes à assumer pour les praticiens et les patients. Nous le vivons actuellement à travers la tragédie de l'épidémie du virus Ebola, où le caractère exceptionnel de la crise internationale s'identifie d'autant plus à une période de guerre où l'ennemi est de l'ordre de l'intime et invisible. Audelà des aspects inhérents au contexte d'expérimentation de nouvelles thérapeutiques, il est difficile de trouver les mesures politiques adéquates. Les différentes logiques de prises en charge des territoires touchés par le virus ou susceptibles de l'être nous incitent à revenir sur les fondements éthiques de nos politiques de santé. Quatre axes sont traditionnellement identifiés dans la littérature. Tout d'abord, le principe de justice qui correspond à la solidarité inter-peuples. Il impose une co-responsabilité et une aide envers les plus vulnérables (rôle des ONG, appuis logistiques, moyens humains et médicaux); il s'oppose à des logiques économiques exclusives portées par des industries pharmaceutiques et interroge les prises de décisions en santé. Le deuxième est le principe de non-malfaisance qui consiste à ne pas nuire aux populations. Suivant cette logique, l'OMS remplit son rôle de protection des populations ciblées en apportant ses ressources. Les gouvernements mettent en place des mesures de prévention, de protection de leurs citoyens (veille sanitaire et mesures mises en place par le ministère de la Santé et les hôpitaux). Le troisième principe est la bienveillance qui invite à respecter Autrui et à poser l'égale dignité entre chaque être humain. Il impose des actions envers ses compatriotes (l'exemple du rapatriement; avec les risques de propagation inter-pays que cela peut engendrer comme l'infection d'une aide-soignante espagnole suite aux soins prodigués sur un patient contaminé) et les personnes infectées. Le quatrième principe est l'autonomie de toute personne de disposer d'un accès aux soins lui permettant une prise en charge globale [2]. 
En ces périodes politiques «exceptionnelles», des tensions éthiques sont palpables quelle que soit l'échelle concernée locale (un hôpital), nationale (pays), internationale (une communauté). Les politiques de gestion de crise nationale, européenne ou internationale montre que cette philosophie utilitariste de la médecine moderne est un mal nécessaire. La diversité du tri trouve d'autres expressions comme l'adéquation délicate entre les moyens économiques et les besoins de santé des populations, visible au quotidien dans nos établissements sanitaires et médicosociaux.

\section{Forger une éthique du tri : entre poids moral et devoir politique}

Trier est un fardeau moral au sens de porter un poids émotionnel, en raison des difficultés techniques et éthiques qui posent les choix à faire comme on l'a vu précédemment avec Ebola, en raison des incertitudes sur leurs conséquences et des contraintes qui font du tri une nécessité. Pourtant, le tri est souhaitable et synonyme de progrès scientifique, la mise en place de protocoles de tri dans la médecine civile fut consubstantielle d'avancées techniques dans le domaine de la dialyse ou en recherche pharmaceutique. Dans le domaine du don et de la greffe d'organes, des progrès nécessaires ont été réalisés dans ce sens et non sans sacrifice. Actuellement en débat, un nouveau mode de prélèvement d'organes dit de classification Maastricht 3: le prélèvement d'organes chez les personnes décédées des suites d'un arrêt cardiaque après une limitation ou un arrêt des thérapeutiques est rendu possible depuis 2005 (décret n²005-949 du 2 août 2005 relatif aux conditions de prélèvement des organes, des tissus et des cellules) et s'inscrit comme mesure de santé publique dans le second plan Greffe lancé au printemps 2012, dont le pilotage est confié à l'agence de la biomédecine. Les nouvelles sources de prélèvements d'organes rendues possibles par la classification Maastricht 3 définissent une voie prometteuse pour pallier le manque de greffons et a déjà fait ses preuves dans d'autres pays tels que les États-Unis ou le Canada. Néanmoins, cette mesure de santé publique fait ressortir de nouveaux enjeux éthiques au niveau français en raison de l'état des connaissances biologiques et du cadre juridique dans lequel le décret s'inscrit. Tout d'abord, elle questionne les critères définitionnels biologiques et culturels de la mort (qu'est-ce que la mort neuronale? Comment définir les altérations des facultés cognitives, les états de conscience? Peut-on imaginer un neurodroit évoluant au rythme des découvertes en neurosciences?). Corréler l'évaluation technique de la mort neuronale, comprise comme la mort de la conscience, à l'établissement de protocoles de prélèvements d'organes soulève la question de la spécificité de chacune des situations rencontrées, le caractère unique de chaque personne. Depuis 2005, la loi Léonetti autorise la limitation et l'arrêt des traitements thérapeutiques chez des patients en fin de vie (sous procédure collégiale réalisée en accord avec la volonté du malade ou de ses proches). La philosophie de la Loi Léonetti, basée sur une concertation entre le patient, l'entourage et l'équipe, est de facto éloignée de l'approche clinique et culturelle des situations de prélèvements d'organes de la catégorie 3 de Maastricht. À l'heure d'une révision de la loi
Léonetti relative aux droits des patients et de la fin de vie, s'interrogeant sur les meilleures conditions de réalisation de procédures de limitations et d'arrêts de traitements actifs suite à la délibération collégiale. On retrouve ici un paradoxe éthique: quand le don de soi et le morcellement de la personne (d'une médecine d'organes évoluant vers une éthique des organes) se subliment en geste de solidarité [3]. «Rule of rescue» jusqu'à quel point? Comment éviter toute dérive économique d'une éventuelle gestion des corps? [4] La mise en place de ce mode de prélèvements d'organes, en France, n'est pas sans questionnement économique. Ce mode de prélèvement ne risque-t-il pas d'affaiblir ceux mis en place? Comment évaluer le coût de gestion économique mis en place en termes de ressources organisationnelles?

\section{Glisser d'une éthique du triage vers un rééquilibrage des inégalités d'accès aux soins}

Comment substituer une logique égalitariste (consistant à pallier directement aux inégalités vécues par des catégories de population) à une logique utilitariste? Le triage est à la fois une promesse et une épreuve: un fondement de l'identité et de la profession médicale moderne et une intrusion menaçante des contraintes économiques et politiques dans la décision médicale. Prenons, en exemple, le rapport Claeys-Léonetti et le texte de la proposition de loi créant de nouveaux droits en faveur des malades et des personnes en fin de vie, le premier axe rappelle les inégalités économiques des citoyens même face à la mort: " $A u$ moment de la mort, nos concitoyens ne sont pas tous égaux. Cette situation dénoncée par de nombreuses études nous indigne et indigne les Français. Les inégalités des citoyens concernant la mort sont particulièrement visibles quand on examine les conditions d'accès aux soins palliatifs » [5].

Face aux difficultés économiques prégnantes dans nos sociétés d'assurer au mieux en fonction des possibilités économiques (nos sociétés sont ruinées !) un égal accès aux soins et des chances optimale de survie, les médecins doivent malgré tout assumer en responsabilités, ici ce qui est le mieux pour le patient et ici dans cet exemple, éviter le «mal mourir». On appose donc à «la bonne mort» «le mal mourir», trouver un équilibre entre le souhait de la personne, les possibilités matérielles, l'arsenal réglementaire et l'accompagnement des familles. Les médecins ne seraient-ils pas des funambules?

Malgré des aspirations égalitaristes visant à promouvoir le plus grand bien et à résorber les inégalités, il semble inéluctable de fabriquer des médecines à vitesse différentes et des rangs de priorisation entre les personnes traduisant ces inégalités. Le tri est «opérant» ou «inopérant » selon le contexte, en situation d'urgence vitale ou pas, par exemple. On peut réfléchir à une approche de type «éthique empirique» du triage en situation. Un exemple, les situations singulières de personnes en extrême situation de précarité sans couverture sociale et soignées dans les Permanences d'accès aux soins en santé (PASS) [16]. 


\section{État-providence, État-nation et gestion des politiques de santé}

\section{Tension éthique et choix économiques}

Tension éthique fondamentale du triage envisagé comme un acte médical à la fois indispensable et insupportable, qui sauve et sacrifie des vies même entre ceux qui ont la chance d'être greffés alors que d'autres restent en liste d'attente, ceux qui ont accès à des lits de soins palliatifs et d'autres personnes âgées qui meurent aux urgences.

Le tri semble montrer que toutes les vies ne se valent pas, ce qui au regard des fondements mêmes de l'Étatnation et de l'État-providence est de l'ordre de l'impensable car garants de nos sécurités et des fondements libéraux de nos sociétés et en termes d'intégrité des états envers leurs citoyens. Selon Ronald Dworkin : "L'intégrité exige que l'[État] parle d'une seule voix, qu'il agisse d'une manière [principielle] et cohérente envers tous [ses] citoyens, qu'il étende à tous les normes [substantielles] de justice ou d'équité qu'il utilise pour certains [6]. L'État, dans sa légitimité, doit conserver une proportionnalité de la justice distributive entre les décisions prises et leurs implications. Dans le tri militaire, il n'est pas sûr que ce soit parce que les vies ne se valent pas qu'ils fassent faire un tri. Bien au contraire, parce que toutes les vies se valent, que face au principe de réalité, la capacité de ne pas pouvoir sauver tout le monde en même temps, ou aussi parce que prendre en charge une personne malade assurément en fin de vie au détriment de plusieurs autres en phase de traitements amènent à accompagner l'un en sa fin de vie (comme en soins palliatifs) alors que d'autres seront pris dans un protocole.

Selon Hans Kelsen : «À ce point de vue, le droit apparaît comme un ordre social fondé sur la contrainte, comme un système de normes prescrivant ou autorisant des actes de contrainte sous la forme de sanctions socialement organisées» [7]. Or, selon Kelsen, un tel système normatif ne résulte pas seulement de l'action du législateur mais également de celle du pouvoir exécutif et judiciaire. De fait, si l'on se réfère à la théorie de la formation du droit par degrés de Kelsen, il n'y a pas de différence de nature entre l'acte de légiférer et celui de juger mais une simple différence de degré : «Constitution, loi, règlement, acte administratif et jugement, acte d'exécution sont simplement les stades typiques de la formation de la volonté collective dans l'État moderne» [8]. Ceci revient à dire que les rôles du constituant, du législateur, du ministre, du juge et plus largement du fonctionnaire ne sont pas fondamentalement différents. Tous s'emploient à produire des énoncés normatifs reposant sur la sanction. Cependant, à mesure que l'on descend dans la hiérarchie normative, les normes perdent leur portée générale (du texte de la constitution politique) pour devenir individuelles (normes dérivant en valeurs juridiques et morales) ${ }^{1}$ [8]. L'assemblée constituante produit des normes générales, le juge produit des normes individuelles. En

\footnotetext{
${ }^{1}$ Hans Kelsen [8], p. 224: «Pour autant que l'on puisse les distinguer, la différence entre la fonction juridictionnelle et la fonction législative consiste avant tout en ce que celle-ci crée des normes
}

outre, plus on descend dans l'échelle normative, plus le degré de création diminue tandis que la part d'application du droit augmente. Ceci implique que la «séparation des pouvoirs» soit une expression trompeuse. Il n'y a pas, à proprement parler, de différence entre les fonctions législative, exécutive et judiciaire. Ce détour par le droit constitutionnel nous montre que nous sommes inscrits dans une logique normative détenues par plusieurs parties prenantes et que le vivant tend à être aussi absorbé par cette logique institutionnelle normative, lui donnant paradoxalement une valeur et donc un coût.

\section{Certifier le vivant: produire des politiques du vivant, de l'éthique, de l'économie?}

Instruire des politiques du vivant, c'est vouloir le faire entrer dans la catégorie d'objet, or précisément «le vivant c'est ce qui résiste». Circonscrire le vivant (on parle de biométrie?) c'est donner au politique la possibilité d'une certification politique du vivant, de poursuivre sa course au normativisme en légiférant sur chaque élément vivant, en transformant des faits biologiques en indicateurs politiques. Un exemple: comment la personne perçoit sa qualité de vie? Nous évaluons le degré de qualité de vie, nous nous évaluons, nous associons donc une valeur à notre personne. Cette approche définitionnelle de la qualité de la vie répond à des contextes socio-historiques (la qualité de vie comme un dispositif historique), à des intérêts cliniques (évaluer le confort de la vie subjective du patient au-delà des symptômes [15], à des perspectives de comparaisons culturelles dans le domaine de la santé mais répond aussi à une problématique épistémologique de la différence entre la vie artificielle et la vie naturelle.

La communauté scientifique est interpellée, preuve en est la montée en puissance des recherches en la matière. Si on regarde l'occurrence des termes de «qualité de vie», dans la littérature internationale, on observe une forte préoccupation depuis les années 1970 et de manière plus marqué sur certains pays comme les États-Unis ou le Canada. La valeur de la vie, l'inutilité (également un concept économique de base) ou la futilité, termes employés à l'égard des traitements médicaux deviennent des indicateurs de la qualité de vie et une réalité économique de prise en charge. Désigner des relations entre la qualité de la vie, la qualité de la fin de vie et la valeur de la vie relève de l'ordre de la souffrance, d'une justification morale de moyens techniques dévolus à une bonne mort et n'est pas sans questionnement économique autour de son évaluation globale de politique de santé.

L'expansion de recherche sur ces concepts dans la littérature manifeste un intérêt grandissant pour comprendre les limites de la qualité de vie et justifier des discours médicaux et politiques du type «au nom de quoi ». Le risque de dérive est de décentrer la décision à l'extérieur du domaine médical et de son expertise. Dans cette perspective, la priorité peut être donnée aux individus qui ont le moins besoin de soins, ou selon un principe d'utilité médicale, à ceux dont les traitements ont les bénéfices les plus probables en termes de

générales, tandis que celle-là ne crée que des normes individuelles». 
durée et de qualité de vie. On peut aussi donner la priorité aux individus que les circonstances rendent indispensables (par exemple, les soignants en situation de catastrophes sanitaires), à ceux ayant des personnes à charge, ou ceux ayant la plus grande valeur sociale ou utilité.

\section{Comment évaluer l'utilité sociale et justifier nos prises de décisions économiques dans des circonstances changeantes?}

Des dilemmes éthiques sont alors inévitables : comment évaluer l'utilité sociale dans des circonstances changeantes? Caractère contingent de l'évaluation de l'individu en rapport à son utilité sociale s'oppose au caractère inviolable de la vie et introduit l'idée d'une valeur de la vie. Quels actions et comportements faut-il suivre? Évaluer les mérites passés d'une vie ou les performances futures d'une vie à mener? Les critères de l'utilité médicale et du pronostic désavantagent les patients les plus graves et les plus âgés. La question de la maximisation de la durée de la vie est épineuse: une année supplémentaire de vie doit-elle être donnée à une personne jeune ou une personne âgée? La visée égalitariste est indispensable tant à la moralité qu'à l'efficacité du triage: l'utilisation optimale des ressources demande d'adopter avec tous un comportement standardisé et impartial, qui minimise les effets d'inégalités et de discriminations.

Cette conception utilitariste est critiquée par la conception égalitariste défendant la restauration de l'accès aux soins pour tous. La visée égalitariste ici basée sur le principe d'équité dont le principe est de remettre à niveau les personnes inégales entre elles, est indispensable à la moralité.

Affirmation égalitariste qui peut aller jusqu'au rejet du triage au nom du respect de la vie humaine (lorsque l'alternative est la vie ou la mort, les êtres humains doivent être considérés comme égaux) et reprendre la conception que toute personne doit être sauvée. Un principe essentiel de l'utilitarisme demande de consacrer le plus de ressources à ceux qui en ont médicalement le plus besoin (services d'urgence et de transplantation).

\section{La progression des droits des patients comme signe d'une démocratie plus participative que représentative}

À cette logique utilitaire (nos sociétés sont en pleine crise économique) et utilitariste (maximiser le plus grand bien pour un plus grand nombre quitte à sacrifier quelques individus) s'oppose une vision de la justice libérale de type rawlsienne mobilisant la coopération patient-médecin (très clairement marquée en recherche biomédicale), la concertation entre les parties prenantes (identifiable notamment lors de la concertation en procédure collégiale) le maintien de la décision souveraine du médecin responsable du patient (serment d'Hippocrate garant de tout conflit d'intérêt, promesse d'une décision médicale rationnelle et égalitaire), affranchie des déterminations sociales de la valeur différentielle des vies et l'égale répartition des chances données à un individu dans une société. Selon le principe de différence de Ralws, les inégalités économiques et sociales doivent procurer le plus grand bénéfice aux membres les plus défavorisés de la société. Ce principe a pour rôle fondamental de répartir les biens entre les membres de la société, de façon à ne laisser personne de côté, et cela, de manière juste et équitable. C'est pourquoi admettre les inégalités n'est pas une mauvaise chose en soi, à condition que cela permette de maximiser le bien des plus défavorisés.

\section{Créer de nouveaux droits des patients: le caractère contraignant des directives anticipées}

L'égalité des chances parfois apparaît sous des atours peu communs. Nous pouvons prendre en exemple la vision philosophique et institutionnelle de Jean-Claude Ameisen sur le rôle des directives anticipées. La proposition de JeanClaude Ameisen, Président actuel du CCNE, est de les rendre contraignantes, replacé dans le contexte philosophique de sa réflexion, elle s'explique en partie par le fait que notre présent est notre futur anticipé: «Notre vie intérieure, notre en nous, cette sensation permanente que ce qui nous arrive, c'est à nous que cela arrive -que ce que nous vivons - c'est nous qui le vivons - fait appel à d'autres temporalités que notre confrontation immédiate au monde extérieur. Il y a en nous un temps intérieur qui s'écoule sans fin» [9]. Alors, on entend dans cette conception l'idée qu'une directive anticipée serait peut-être le côté tangible d'un futur par essence maîtrisé et peut-être une expérience de mort ou de vie imminente nous poussant à anticiper. L'idée de les rendre contraignante revient à poser une coresponsabilité entre le patient et le médecin, le médecin ne détenant plus seul la responsabilité finale. On observe une progression forte du libéralisme des droits des patients à travers cette proposition.

\section{Une philosophie médicale du tri institutionnalisé par opposition au champ ouvert et libre de la réflexion éthique}

Ce double mouvement économique libéral-égalitariste fait du «triage» le pivot de débats plus généraux, qui ont trait d'une part, au choix, en amont des pratiques médicales et des populations sur lesquelles concentrer les ressources collectives de santé (la macro-allocation visible, notamment dans la prise en charge des maladies dites chroniques, diabète, insuffisance rénale, etc...) et, d'autre part, aux critères éthiques et médicaux servant à définir l'accès des patients individuels à des ressources rares (la microallocation, recherches sur les maladies rares et orphelines, par exemple). Une telle extension ouvre la question sur l'idée d'un continuum contemporain du tri par opposition au champ de l'éthique qui refuse de s'inscrire dans un continuum institutionnel: "L'éthique conceptuelle ${ }^{2}$, cette haute idée que l'on se fait d'un idéal - cherche à être apprivoisé par l'individu et le collectif - l'organisation sociale

\footnotetext{
${ }^{2}$ Nous proposons ici d'inventer ce concept d' 'éthique conceptuelle» pour désigner la conception idéaliste qui est faite de l'éthique, de nos jours.
} 
et politique n'a de cesse que de chercher à contrôler ce qui a priori (mais nous n'en avons aucune certitude in fine) relève du domaine de l'éthique: le corps, l'âme, le sujet, l'individu, l'intime. L'éthique, bien que conceptuelle, ne semble pas être passée au stade de l'institutionnalisation, et c'est bien cet état ici que nous questionnons à travers la question du droit de mourir [10] car s'y inscrivant, elle permet toute sa liberté de déranger, de questionner, de reconnaître les personnes dans leurs individualités et citoyenneté.

Dans le champ du handicap, le rapport de Pascal Jacob sur l'accès aux soins et à la santé des personnes en situation de handicap montre bien qu'en refusant de reconnaître la citoyenneté des personnes, on les prive délibérément d'accès à des ressources: «Proposition 19. Inscrire une exigence de reconnaissance, d'accueil et de considération de la personne en situation de handicap et de son "Référent du Parcours de Santé» dans les relations avec l'hôpital (urgences, consultation, hospitalisation) dans le cadre de la certification des établissements de santé par la Haute Autorité de santé (HAS), 30. Mettre en place un dispositif d'identification des personnes handicapées lors d'un appel aux services d'urgences, pompiers, samu...». Reconnaître la citoyenneté des individus, c'est les insérer dans un parcours de soins et les reconnaître dans leurs identités personnelles».

«Faire vivre et laisser mourir» [17]: utiliser la notion de tri permet de souligner comment des configurations politiques parfois consensuelles tracent une ligne de partage entre ceux qu'il s'agit de «faire vivre » et ceux qu'il «s'agit de laisser mourir», si bien que le droit à la sédation profonde en tant que droit du patient interpelle: «Le droit à la sédation profonde et continue jusqu'au décès en phase terminale est créé. Selon le CCNE, dans son avis 121, «il s'agirait d'un droit nouveau qui viendrait s'ajouter au droit de refuser tout traitement et au droit de se voir prodiguer des soins palliatifs quand ceux-ci sont indiqués", en réponse au constat partagé sur le «mal-mourir» en France». "Cette sédation doit répondre à la demande légitime des personnes atteintes d'une maladie grave et incurable qui envisagent leur fin de vie, de ne pas souffrir et ne pas prolonger, selon elles, inutilement cette période ultime. Nous souhaitons leur donner l'assurance de bénéficier d'un traitement à visée sédative et antalgique provoquant une altération profonde et continue de la vigilance jusqu'au décès associé à l'arrêt de l'ensemble des traitements de maintien en vie». La sédation est profonde pour garantir l'altération totale de la conscience, cet endormissement prévenant toute souffrance, y compris celle résultant de «se voir mourir». Selon les termes de Jean-Claude Ameisen, lors de son audition, «je ne suis pas obligé d'être le témoin de ce qui va advenir».

L'éthique du triage consiste dès lors à identifier, hiérarchiser et agencer les critères de priorisation en fonction des principes et valeurs jugées préférables; il s'agit d'articuler et de donner le poids le plus juste à des principes contradictoires car le triage est traversé par une tension entre utilitarisme et égalitarisme. Il s'inscrit dans une morale utilitariste, prescrivant de sauver ou soigner le plus grand nombre de patients et une morale égalitariste prescrivant de les traiter de manière équitable. Et même dans le domaine de la gestion de la fin de vie, le manque de ressources allouées aux soins palliatifs est considéré comme une perte de chance d'être traité dignement à la fin de sa vie. Les principes utilitaristes mis en jeu dans le triage orientent vers des stratégies de maximisation du bien, par exemple du nombre de vies ou d'années de vies sauvées, ou du bonheur social total et s'oppose en cela à des visions très libérales (éthique minimale de Rugen Ogien) ou libertarienne (comme le défend Robert Nozick) ou les droits individuels (dont la libre disposition du corps) prime sur la liberté collective et où seule la vision de sécurité de soi-même ou autrui autorise le droit d'ingérence de l'État-Léviathan: «La seule raison légitime que puisse avoir une communauté pour user de la force contre un de ses membres est de l'empêcher de nuire aux autres (...). Sur lui-même, sur son corps et son esprit, l'individu est souverain» [11].

\section{Faire au mieux : progresser dans le domaine de la recherche afin de rendre adéquat les biens de la santé aux besoins évalués}

Pour Michael Walzer, les biens de la santé doivent être distribués par le critère du besoin, à même besoin doit être proposés les mêmes soins. La recherche sur l'homme n'est pas un bien en elle-même, mais elle est un moyen nécessaire pour offrir des soins meilleurs (ce qui est un bien). Il importe donc que les résultats de la recherche soient utiles à tous ceux qui sont atteints d'une pathologie donnée, ce qui suppose que les participants à la recherche soient représentatifs des personnes susceptibles de bénéficier de ses résultats. Ici à même besoin, les patients doivent avoir un même accès aux soins et donc aux recherches.

Éviter la normalisation des pratiques (EBM), promouvoir la singularité, c'est faire vivre la logique d'une justice distributive. La justice distributive fait intervenir une notion de proportionnalité dans la répartition des biens dans la mesure où il n'existe pas de parfaite égalité entre les individus et que les besoins diffèrent d'un individu à l'autre. Plusieurs approches ont été proposées pour concevoir la répartition des «biens médicaux» entre les individus: les approches utilitaristes, égalitariste et prioritariste.

Ces trois approches reposent sur différents principes: le principe «sauvant le plus de vies», le principe du «pronostic en années de vie» de valeur d'utilité sociale (pour les approches utilitaristes), le principe de loterie du «premier arrivé, premier servi» ou encore le principe de réciprocité (pour les égalitaristes), principe de la priorité aux plus malades, ou au plus jeune (pour les prioritaristes). Un des exemples d'utilitarisme est celui de Engelhardt qui considère qu'une réflexion sur les allocations de ressources rares et donc sur les critères de triage, est indispensable pour diminuer l'inégalité d'une éventuelle loterie naturelle.

Engelhardt propose un index de droit au traitement (Treatment Entiltlement Index) calculé pour chaque patient prenant en compte les chances de succès au traitement, la survie, la qualité de vie de l'individu proposé au service de réanimation. Selon l'accessibilité de la ressource, l'index du seuil d'admission varierait. Ce choix est guidé par le «meilleur intérêt» pour le patient, mais aussi par la volonté d'éviter une perte de chance pour d'autres patients et de prendre en compte le coût de la prise en charge [1]. 
Chaque approche est critiquable, avec des avantages et des inconvénients qui expliquent leurs limites et leur faible utilisation, soit elles tendent à stigmatiser les individus a priori et de manière collective (approche utilitariste), soit elles ne permettent pas une réelle justice dans la répartition des ressources en ne prenant en compte qu'un facteur parmi beaucoup d'autres (égalitarisme et prioritarisme). D'autres théories de justice sont déployées : Persad et al. [1] recommandent un modèle de vie globale construit avec 4 à 6 des 8 principes des trois théories essentielles comportant plus de critères pronostiques, mais a tendance à défavoriser les patients les plus âgés car un des principes valorise le plus jeune. Scheunemann et White [12] proposent 4 critères permettant une certaine moralité des décisions: une surveillance des décisions par une institution légitime, un processus décisionnel permanent, un raisonnement fondé sur des informations et des principes cohérents et acceptés par tous et un dispositif permettant aux personnes concernées de faire appel des décisions.

\section{De la quantification de mesures du vivant à la solidarité humaine}

Quantifier et trier le vivant répond à une demande de certification politique, mais aussi aide à élaborer des politiques du bien-être, différant d'une personne à une autre. Promouvoir un consensus par recoupement, comme chez Rawls, c'est espérer avoir un pluralisme de conceptions de la santé bonne pour chacun et compatibles avec le collectif. Le libéralisme politique se fonde sur un pluralisme raisonnable qui prend en compte une pluralité de doctrines compréhensives, religieuses ou non. Ce pluralisme est vu comme le résultat de l'activité naturelle de la raison humaine quand elle se déroule dans un contexte durable d'institutions libres. Selon Rawls : «le succès du constitutionnalisme libéral est apparu comme la découverte d'une nouvelle possibilité sociale : la possibilité d'une société pluraliste relativement stable et harmonieuse» [13].

Dans Libéralisme politique, Rawls montre que la délibération avant d'être collective est individuelle. En effet, l'interrogation première de Rawls est «comment est-il possible que se perpétue une société stable et juste, constituée de citoyens libres et égaux, mais profondément divisés entre eux en raison de leurs doctrines compréhensives, morales, philosophiques et religieuses, incompatibles entre elles bien que raisonnables?».

La théorie de Ronald Dworkin va précisément dans ce sens. Selon cet auteur, il faut distinguer deux types de questions qui appellent des formes de réponse différentes. Ainsi, il conviendrait de distinguer entre les questions politiques et de principes. Les «questions politiques» qui portent sur le bien-être de la collectivité politique et doivent être tranchées au moyen «d'arguments utilitaires». Les «questions de principes » qui sont d'ordre éthique et font entrer en ligne de compte des impératifs catégoriques que l'on ne saurait transgresser [14].

Le besoin impératif de sauver une vie suppose une préférence pour des mesures qui sauvent par rapport à celles qui ne sauvent pas et entre dans le champ des «questions de principes » de Dworkin.
Le devoir de sauver intervenant en situation inattendue, extraordinaire, exceptionnelle, ne prend en compte ni le coût, ni le bénéfice attendu, notamment en termes de durée de vie. La réponse est immédiate et ne s'inscrit pas dans le temps, au contraire des actions médicales fondées sur une analyse coût-utilité. Le devoir de sauver apparaît en conflit avec les stratégies rationnelles. Ce devoir de secours mobilise les citoyens d'une communauté et a une certaine utilité sociale de rassemblement autour de valeurs et entre du coup dans le champ des questions politiques. L'évaluation de la performance d'un système de santé et de ses moyens où il existe une pluralité de valeurs peut tromper la rationalité utilitariste, car nous superposons aux questions politiques du vivre ensemble les questions de principes qui se tranchent au cas par cas.

\section{Conclusion}

La démocratie peut-elle se décliner avec ou sans le peuple? La crise et la mutation de l'expérience démocratique se joue-t-elle entre une médecine du tri et médecine personnalisée?

Doit-on considérer que le peuple est, avant tout une «communauté morale» sans laquelle la «communauté légale» perdrait tout son fondement? Sur le plan théorique et philosophique, un effort des politiques publiques à atténuer ou à remédier à la perte de confiance dans les liens de citoyenneté est visible notamment en ce qui concerne le champ de la santé ou de l'éthique ou des modes de consultation populaire sont favorisés.

Mélange de démocratie participative et représentative, qui permet aux citoyens de se faire entendre. L'ensemble des pratiques, des attitudes et des comportements politiques ressortissant à l'expérience contemporaine d'une «citoyenneté critique» et reliés à des mouvements plus profonds de transformation critique de la démocratie.

Système libéral en crise qui propose comme solution une participation citoyenne plus forte (hégémonie des droits individuels comme nous l'avons vu en termes de droits des patients), ce que Habermas énonce comme une nécessité de porter la citoyenneté démocratique au-delà de la nation. On parle dès lors de médecine personnalisée.

\section{Déclaration d'intérêts}

L'auteur déclare ne pas avoir de conflits d'intérêts en relation avec cet article.

\section{Références}

[1] Lachenal G, Lefeve C, Vinh-Kim N. La médecine du tri. Histoire, éthique, anthropologie. Paris: Presses universitaires de France; 2014.

[2] Brehaux K, Ferriere F, De Champs C, Andreoletti L, Tournay V, Vezy B. Ebola, émergence en Afrique de l'Ouest : histoire d'une épidémie imprévisible? Paris: Le Quotidien du Médecin; 2014.

[3] Brehaux K, Tournay V. Quand la santé publique rencontre la bioéthique. Comment concilier éthique de fin de vie et prélèvements d’organes? Paris: Le Quotidien du médecin; 2014. 
[4] Boles JM. Les prélèvements d'organes sur donneur décédé après arrêt cardiaque. Tome 58. Paris: Revue Laennec; 2010.

[5] Claeys A, Leonetti J. http://www.elysee.fr/assets/Uploads/ Rapport-et-proposition-de-loi-creant-de-nouveaux-droits-enfaveur-des-malades-et-des-personnes-en-fin-de-vie.pdf

[6] Dworkin R. (1986), Empire des lois. (1986), trad. Elisabeth Soubrenie. Paris: Presses universitaires de France; 1994.

[7] Kelsens H. Théorie pure du droit. Neuchâtel (Suisse): La Baconnière; 1988. p. 71.

[8] H. Kelsen, «La garantie juridictionnelle de la Constitution (La justice constitutionnelle)», R.D.P, 1928, no. 35, p. 199.

[9] Ameisen JC. Sur les épaules de Darwin, les battements du temps. Paris: Éditions Poche; 2014. p. 66.

[10] Brehaux K. Quelle place pour les droits individuels et les libertés publiques? La question de l'euthanasie aujourd'hui en France. Paris: Communication Cevipof-CNRS; 2014.

[11] Stuart Mill J. De la liberté. Paris: Presses Universitaires de France; 1990.
[12] Scheunemann LP, White DB. The ethics and reality of rationing in medicine. Chest 2011;140(6):1625-32.

[13] Rawls J. Libéralisme politique. Paris: Presses Universitaires de France; 1995. p. 13.

[14] Dworkin R. Une question de principe. Paris: Presses universitaires de France; 1996. p. 3.

[15] Ferrans C, Powers JM. L'indice de qualité de vie: développement et propriétés psychométriques, 88. Paris: Association de recherche en soins infirmiers; 2007. p. 32-7.

[16] Georges C, Tapie de Celeyran, Platon FJ, et al. Décider en cancérologie dans les situations médicosociales complexes: les réunions de concertation pluriprofessionnelles médicosociales et éthiques à l'hôpital Saint-Louis de Paris. Oncologie 2014;16:55-62.

[17] Memmi D. Faire vivre et laisser mourir: le gouvernement contemporain de la naissance et de la mort. Paris: La Découverte; 2003. 